\title{
Effect of superconducting fluctuations on ultrasound in unconventional superconductor
}

\author{
M.S. Mar'enko ${ }^{1}$, C. Bourbonnais ${ }^{1,2}$, and A.-M.S. Tremblay ${ }^{1,2}$ \\ ${ }^{1}$ Département de physique and Regroupement québécois sur les matériaux de pointe, \\ Université de Sherbrooke, Sherbrooke, Québec, J1K 2R1, Canada \\ ${ }^{2}$ Institut canadien de recherches avancées, Université de Sherbrooke, Sherbrooke, Québec, J1K 2R1, Canada
}

(Dated: November 15, 2018)

\begin{abstract}
We study the renormalization of sound attenuation and sound velocity by fluctuation Cooper pairs in layered superconductors. We consider the influence of $s$ - and $d$-wave symmetry of the fluctuating order parameter, on both longitudinal and transverse phonon modes. We show that both unconventional order parameter symmetry and transverse sound polarization suppress the AL and MT terms, while the DOS contribution is the least affected. The combination of these effects can change the sign of the overall fluctuation corrections above $T_{c}$. We also compare the results obtained using the Ginzburg-Landau formalism with a microscopic derivation of the fluctuation corrections to the sound velocity in both $s$ - and $d$-wave superconductors. These calculations are motivated by ongoing ultrasound measurements in organic superconductors.
\end{abstract}

PACS numbers: 74.25.Ld, 74.25.Fy, 74.40.+k

\section{INTRODUCTION}

Ultrasound is a very powerful tool to study the electronic properties of metals. In systems such as $\mathrm{Sr}_{2} \mathrm{RuO}_{4}$, [1] heavy fermions, [2] and organic superconductors [3] the ultrasound technique was used to probe the symmetry of the order parameter. In layered organic superconductors, ultrasound attenuation was one of the key methods to determine the phase diagram. 3] Compounds from the $\kappa$-(ET) $)_{2}$-X family can exist in different phases, such as normal metal, superconductor or Mott insulator. Most phase transition lines can be associated with anomalies of the sound velocity and sound attenuation as a function of temperature or pressure. [3] In particular, preliminary experiments indicate that the N-S phase transition is accompanied by a pronounced fluctuation region. This can be explained as an effect of transient Cooper pairs above $T_{c}$, or, equivalently, fluctuations of the superconducting order parameter. It is known that in layered organic materials the fluctuation region is especially broad due to strong electronic anisotropy, low charge carrier concentration and relatively high $T_{c}$. The manifestation of superconducting fluctuations in various physical phenomena in organic superconductors has been experimentally confirmed. [4, 5]

The sound attenuation and the sound velocity in metals are determined at low enough temperatures by the interaction of phonons with the electronic system. In the Fröhlich model, phonons couple to the electronic density, hence the electron-phonon interaction is momentumindependent. This approach is not valid, [6] however, in many systems of interest. In the presence of impurities, one has to consider the electron system in a reference frame moving together with the ion lattice. 6, 77, 8] Indeed, in an impure metal, the elastic scattering of electrons as well as perfect screening at small phonon momentum and relaxation to equilibrium occur in this oscillating frame. In this formalism, the electron-phonon coupling appears through the stress tensor, rather than through the density operator.

In order to include polarization effects on sound propagating in highly anisotropic media such as organic superconductors, one has to consider a tight-binding model instead of a continuous one. In the tight-binding model, the electron-phonon interaction comes from the modulation of the hopping parameter induced by the lattice deformation when the sound wave propagates through the crystal. The electron-phonon vertex then depends on the orientation of the phonon momentum and the phonon polarization. In such a model, where the stretching of specific crystal bonds induced by the lattice deformation contributes to the electron-phonon vertex, [9] one can explain for example that anisotropy in the attenuation of different phonon modes can be as large as few orders of magnitude in $\mathrm{Sr}_{2} \mathrm{RuO}_{4}$. [1]

In the superconducting state, the symmetry of the order parameter can also affect the temperature dependence of the sound attenuation. Namely, it does depend on the presence of nodes and on their orientation with respect to the phonon momentum and polarization. Thus, sound attenuation experiments can probe the type of superconducting pairing. 10, 11]

One would naturally expect that the superconducting fluctuations 12, 13, 14 will interact with ultrasound in a similar way, thus revealing the order parameter symmetry. The magnitude of the ultrasound renormalization by fluctuations is not necessarily small. Previously, the fluctuation corrections to longitudinal phonon mode propagating perpendicular to the conduction layers in quasi2D material were found assuming $s$-wave symmetry of the energy gap. 15] The model that uses the quasi-2D open Fermi-surface and the electron-phonon vertex originating from the modulation of the electron interlayer hopping integral in the presence of the ultrasound wave predicts rounding of the sound attenuation and sound velocity temperature dependencies near $T \rightarrow T_{c}$. 
Since there is a substantial experimental interest in this subject, we present estimations for the fluctuation corrections to the sound attenuation and sound velocity for longitudinal and transverse ultrasound propagating perpendicular to the conduction planes, for $s$ - and $d$-wave symmetries of the order parameter.

The paper is organized as follows: in Section II we present a simple estimation for the fluctuation corrections to the sound velocity that can be obtained from the Ginzburg-Landau formalism in order to provide a simple phenomenological basis for the microscopic results. In Section [II we describe the microscopic model for the quasi-2D superconductor and for the electron-phonon interaction at various sound polarizations. In Section IV we review the sound attenuation and sound velocity in the mean field approximation (without superconducting fluctuations). In Sections $\mathbf{D}-\mathrm{VI}$ we give the superconducting fluctuation corrections for various pairing symmetries and phonon polarizations. Finally, we present a discussion of our results and of their relevance to experiment.

\section{GINZBURG-LANDAU APPROACH TO THE FLUCTUATION SOUND VELOCITY}

In this section, we obtain the fluctuation corrections to the sound velocity from the Ginzburg-Landau free-energy functional. Before that, we shall give, for completeness, a summary of elasticity theory that can be found in more details elsewhere. [16, 17] Even though it is less detailed than the microscopic approach that is given later, the Ginzburg-Landau point of view allows one to develop physical intuition. It can also provide an independent check of the microscopic results that are given in the rest of the paper.

\section{A. Sound velocity jump at $T=T_{c}$}

The difference between superconducting and normal state free energies at temperatures close to $T_{c}$ without fluctuations is

$$
\Delta F_{N S}=F_{N}-F_{S}=\frac{\alpha^{2}\left[T-T_{c}\left(\epsilon_{i j}\right)\right]^{2}}{8 \pi},
$$

where $\alpha$ is the Ginzburg-Landau coefficient, and

$$
\epsilon_{i j}=\frac{1}{2}\left(\frac{\partial u_{i}}{\partial x_{j}}+\frac{\partial u_{j}}{\partial x_{i}}\right)
$$

is the $(3 \times 3)$ strain tensor that is defined through the displacement $\mathbf{u}$ at a point $\mathbf{x}$. In a continuous elastic medium, $\epsilon_{i j}$ obeys Hooke's law:

$$
\sigma_{i j}=c_{i j k l} \epsilon_{k l},
$$

where $\sigma_{i j}$ is the stress tensor and $c_{i j k l}$ the elastic modulus tensor. Symmetry considerations allow us to reduce the number of independent components in Eq. (3), thus lowering the rank of $\epsilon_{i j}$ and $\sigma_{i j}$ and converting them into $(1 \times 6)$ vectors $\epsilon_{m}$ and $\sigma_{m}$ with the following rule that relates the indices $\{i j\}$ and $\{m\}$ :

$x x \rightarrow 1, y y \rightarrow 2, z z \rightarrow 3, y z \rightarrow 4, z x \rightarrow 5, x y \rightarrow 6$.

Similarly, the elastic modulus tensor $c_{i j k l}$ is converted into a symmetric $(6 \times 6)$ tensor $c_{m n}$ according to $j i \rightarrow m$, $k l \rightarrow n$, and the rule (4). The tetragonal system symmetry reduces the number of independent components of $c_{m n}$ to only 6 and imposes additional relations between them: $c_{11}=c_{22}, c_{44}=c_{55}, c_{12}=c_{21}, c_{13}=c_{23}=c_{31}=$ $c_{32}$, and all other off-diagonal components are 0 .

The equation of motion for sound waves results in the relation for the sound velocity $v_{s}$ :

$$
\bar{c}=\rho v_{s}^{2},
$$

where $\rho$ is the density, and $\bar{c}=c_{i j k l} \hat{q}_{i} \hat{q}_{j} \hat{e}_{k} \hat{e}_{l}$ is the contraction of the elastic modulus tensor with the phonon momentum and polarization vectors. In particular, for longitudinal phonons propagating along $z$-axis, the $\bar{c}$ being expressed in terms of the $(6 \times 6)$ tensor is $\bar{c}=c_{33}$. For transverse phonons with the momentum $\widehat{\mathbf{q}} \| \widehat{\mathbf{z}}$ and polarization $\widehat{\mathbf{e}}$ lying in the $x y$ plane, $\bar{c}=c_{13}$ for any angle $\varphi$ between the vector $\widehat{\mathbf{e}}$ and $x$-axis.

Then, with the notations

$$
\Gamma_{m}=\frac{\partial T_{c}}{\partial \epsilon_{m}}, \quad \Delta_{m n}=\frac{\partial^{2} T_{c}}{\partial \epsilon_{m} \partial \epsilon_{n}},
$$

the discontinuity in the elastic modulus tensor at $T=T_{c}$ that is related to the second derivative of $\Delta F_{N S}$ with respect to strain is [18]:

$$
\Delta c_{m n}=\left.\frac{1}{V_{0}} \frac{\partial^{2} \Delta F_{N S}}{\partial \epsilon_{m} \partial \epsilon_{n}}\right|_{T=T_{c}}=\frac{\alpha^{2} \Gamma_{m} \Gamma_{n}}{4 \pi V_{0}},
$$

where $V_{0}$ is the unstrained volume. We shall consider the thermodynamics of the longitudinal phonons only, because for transverse modes $\Gamma_{m}$ vanishes by symmetry hence there is no discontinuity in $c_{m n}$. Using the relation for the sound velocity Eq. (5), one finally obtains the sound velocity jump at $T=T_{c}$ :

$$
\Delta v_{s}=\frac{\Delta \bar{c}}{2 v_{s} \rho}=\frac{\alpha^{2} \overline{\Gamma_{m} \Gamma_{n}}}{8 \pi v_{s} \rho V_{0}},
$$

where the summation in $\overline{\Gamma_{m} \Gamma_{n}}$ is done over all indices $m$ and $n$ that correspond only to non-zero components of $\bar{c}$ for the particular $\widehat{\mathbf{q}}$ and $\widehat{\mathbf{e}}$. Finally, the sound velocity jump can also be related to the specific heat jump at $T=T_{c}$

$$
\Delta C_{N S}=-T_{c} \frac{\alpha^{2}}{4 \pi V_{0}}
$$

as follows: [18]

$$
\frac{\Delta v_{s}}{v_{s}}=-\frac{\Delta C_{N S}}{T_{c}} \frac{1}{2 \rho} \overline{\left(\frac{1}{v_{s}} \frac{\partial T_{c}}{\partial \epsilon_{m}}\right)\left(\frac{1}{v_{s}} \frac{\partial T_{c}}{\partial \epsilon_{n}}\right) .}
$$


This relation is quite general. It holds for both isotropic and anisotropic materials in the mean field approximation.

\section{B. Fluctuation corrections to the sound velocity at $T>T_{c}$ in the $3 \mathrm{D}$ isotropic case}

We can use the Ginzburg-Landau formalism to obtain the corrections to the sound velocity at $T>T_{c}$. In the presence of superconducting fluctuations, the free energy acquires the term :

$$
F_{\mathrm{fl}}=-T \sum_{p<p_{0}} \ln \frac{\pi}{\alpha\left(\epsilon+\eta_{c} p^{2}\right)}
$$

where $\epsilon=\left(T-T_{c}\right) / T, \eta_{c}=1 /(4 m \alpha T)$, and $p_{0} \sim 1 / \sqrt{\eta_{c}}$ is a cut-off. Then the most singular contributions are:

$$
\frac{\partial^{2} F_{\mathrm{fl}}}{\partial \epsilon_{m} \partial \epsilon_{n}}=-\sum_{p<p_{0}}\left[\frac{\Gamma_{m} \Gamma_{n}}{T\left(\epsilon+\eta_{c} p^{2}\right)^{2}}+\frac{\Delta_{m n}}{\epsilon+\eta_{c} p^{2}}\right] .
$$

Here the structure of the second term resembles the 3D fluctuation propagator $L(\Omega=0, p)$ in the Gaussian fluctuation theory [19], while the first term of Eq. (12) looks like the fluctuation propagator squared.

In Eq. (12), the main contribution is from the first term. If one changes summation to integration and sets the upper limit to infinity, in the limit $\epsilon \rightarrow 0$ it reads:

$$
-\int_{0}^{p_{0}} \frac{\Gamma_{m} \Gamma_{n}}{T\left(\epsilon+\eta_{c} p^{2}\right)^{2}} \frac{2 \pi p^{2}}{(2 \pi)^{3}} d p=-\frac{\Gamma_{m} \Gamma_{n}}{16 \pi T_{c} \eta_{c}^{3 / 2} \sqrt{\epsilon}} .
$$

This expression is singular at $T=T_{c}$ and clearly resembles the standard fluctuation conductivity contribution of the Aslamazov-Larkin type in the 3D case [12]. The second term of Eq. (12) being integrated over momenta $p$ is not singular as $\epsilon \rightarrow 0$.

Thus, considering for simplicity only diagonal terms of the strain tensor, the corrections to the sound velocity are given by:

$$
v_{s f l}=\frac{1}{2 v_{s} \rho} \frac{\partial^{2} F_{\mathrm{fl}}}{\partial \varepsilon_{m} \partial \varepsilon_{m}}=-\frac{\Gamma_{m} \Gamma_{n}}{32 \pi v_{s} \rho T_{c} \eta_{c}^{3 / 2} \sqrt{\epsilon}} .
$$

Note that the standard derivation of the fluctuation corrections to the electronic specific heat, that requires taking second derivative of the thermodynamic potential with respect to temperature, results in the expression [20]

$$
C_{\mathrm{fl}}=\frac{1}{16 \pi \eta_{c}^{3 / 2} \sqrt{\epsilon}}
$$

that is in agreement with Eqs. (10) and (14). The fluctuation corrections to sound velocity and specific heat here are connected in the same way as the mean-field jumps. This is not the case in the Lawrence-Doniach model, as we will discuss in the next section.

\section{Fluctuation corrections in Lawrence-Doniach model}

In the case of layered materials, one should use the Lawrence-Doniach quasi-2D free energy rather than the isotropic 3D one. At a low electron density, the quasiparticle spectrum can be taken to be

$$
\xi\left(p_{\|}, p_{z}\right)=\frac{p_{\|}^{2}-p_{F}^{2}}{2 m}-2 t_{\perp} \cos \left(p_{z} c\right)
$$

where $p_{\|}$denotes the intralayer component of the momentum, $p_{z}$ the interlayer component, $c$ the interlayer distance, and $t_{\perp}$ the interlayer hopping integral. The Fermi surface then has the form of a corrugated cylinder with the one-particle density of states given by $\nu_{0}=$ $m /(2 \pi c)$. Then, the fluctuation term analogous to Eq. (11) reads:

$$
F_{\mathrm{fl}}=-T \sum_{p<p_{0}} \ln \frac{\pi}{\alpha\left\{\epsilon+\eta_{\|} p_{\|}^{2}+\frac{r}{2}\left[1-\cos \left(p_{z} c\right)\right]\right\}} .
$$

Now $\eta_{\|}$is related to the square of the in-plane correlation length, and the parameter $r$ characterizes the material anisotropy. The modulation of hopping integral $\delta t_{\perp}$ by the strain $\epsilon_{m}$ due to the lattice deformation by the sound wave contributes to the electron-phonon interaction, that should be taken into account in the second derivative of $F_{\mathrm{fl}}$ Eq. (17) with respect to strain. Without going into all the details, we assume for the moment simple proportionality relations: $r \propto t_{\perp}^{2}$ and $\delta t_{\perp} \propto\left(\partial t_{\perp} / \partial u_{i}\right)\left(\partial u_{i} / \partial x_{j}\right) \delta x_{j}$, where $\left(\partial t_{\perp} / \partial u_{i}\right)^{\perp}$ contributes to the appropriate electron-phonon coupling constant $g$, and the combination $\left(\partial u_{i} / \partial x_{j}\right)$ can be rewritten in terms of the strain tensor Eq. (2). Therefore, one has:

$$
\begin{aligned}
& \frac{\partial^{2} F_{\mathrm{fl}}}{\partial \epsilon_{m} \partial \epsilon_{n}}=-\frac{1}{T} \int_{0}^{p_{0}} \int_{-\pi / c}^{\pi / c} \frac{d p_{z} p_{\|} d p_{\|}}{(2 \pi)^{2}} \\
& \times \frac{\left\{\Gamma_{m}-g t_{\perp} T\left[1-\cos \left(p_{z} c\right)\right]\right\}\left\{\Gamma_{n}-g t_{\perp} T\left[1-\cos \left(p_{z} c\right)\right]\right\}}{\left\{\epsilon+\eta_{\|} p_{\|}^{2}+\frac{r}{2}\left[1-\cos \left(p_{z} c\right)\right]\right\}^{2}} \\
& -\int_{0}^{p_{0}} \int_{-\pi / c}^{\pi / c} \frac{d p_{z} p_{\|} d p_{\|}}{(2 \pi)^{2}} \frac{\Delta_{m n}-g^{2} T\left[1-\cos \left(p_{z} c\right)\right]}{\epsilon+\eta_{\|} p_{\|}^{2}+\frac{r}{2}\left[1-\cos \left(p_{z} c\right)\right]} .
\end{aligned}
$$

We separate the expression in the last line of Eq. (18) into two parts. The first one, without $\cos \left(p_{z} c\right)$ in the numerator, results in the following contribution to the sound velocity:

$$
-\frac{\nu_{0}\left(\overline{\Delta_{m n}}-g^{2} T\right)}{2 m v_{s} \rho \eta_{\|}} \ln \left(\frac{2}{\sqrt{\epsilon}+\sqrt{\epsilon+r}}\right) .
$$

The other part, with $\cos \left(p_{z} c\right)$ in the numerator, gives a contribution of the type:

$$
-\frac{g^{2} T \nu_{0}}{4 m v_{s} \rho \eta_{\|}} \frac{(\sqrt{\epsilon}-\sqrt{\epsilon+r})^{2}}{r} .
$$


In the integral in the second line of Eq. (18), one can keep the terms in the numerator that do not contain elastic derivatives $\Gamma_{m}$. This leads to a contribution to the sound velocity of the following type:

$$
-\frac{g^{2} T \nu_{0}}{2 m v_{s} \rho \eta_{\|}}\left[1+\frac{2 \epsilon}{r}\left(-1+\sqrt{\frac{\epsilon}{\epsilon+r}}\right)\right] .
$$

Keeping the $\Gamma_{m} \Gamma_{n}$ term in the numerator of the integral in the second line of Eq. (18), and neglecting the hopping terms, gives the contribution:

$$
-\frac{\nu_{0} \overline{\Gamma_{m} \Gamma_{n}}}{4 m v_{s} \rho T_{c} \eta_{\|} \sqrt{\epsilon(\epsilon+r)}} .
$$

Finally, the cross-term that is proportional to both $\Gamma_{m}$ and $g t_{\perp}$, results in the contribution:

$$
-\frac{g \nu_{0} \overline{\left(\Gamma_{m}+\Gamma_{n}\right)}}{2 m v_{s} \rho \eta_{\|}} \frac{(\sqrt{\epsilon}-\sqrt{\epsilon+r})}{\sqrt{r(\epsilon+r)}}
$$

that is less singular than Eq. (22) at small $\epsilon$ and $r$.

Note that the relation (10) between the mean-field jumps of the sound velocity and specific heat at $T=T_{c}$ is satisfied in the Lawrence-Doniach model. This comes about from the fact that in this model the difference between superconducting and normal state free energies is still given by Eq. (11) with the parameters modified for the quasi-2D case. On the other hand, the relation Eq. (10) is not satisfied for the fluctuation corrections in this model. This is because we can neglect any $T$ dependence of the interlayer hopping, hence it does not contribute to the specific heat. We will obtain later in the microscopic calculations in Section $\nabla$ the expressions that have the temperature dependence of Eqs. (19)-21).

\section{MICROSCOPIC MODEL}

\section{A. Quasiparticle energy spectrum}

We use the model of quasi-2D square lattice with the in-plane period $a$, and the interlayer distance $c>a$. The quasiparticle energy spectrum in the normal state in the lattice model is given by:

$$
\begin{aligned}
\xi(\mathbf{p})= & -2 t_{\|}\left[\cos \left(p_{x} a\right)+\cos \left(p_{y} a\right)\right]-\mu \\
& -2 t_{\perp} \cos \left(p_{z} c\right) .
\end{aligned}
$$

Here $t_{\|}$and $t_{\perp}$ are the intralayer and interlayer hopping integrals. In highly anisotropic materials we have $t_{\|} \gg t_{\perp}$, and the electrons are moving preferentially in the conduction layers. (In organic materials such as $\kappa$ $(\mathrm{ET})_{2} \mathrm{Cu}(\mathrm{NCS})_{2}$, for example, we can estimate the ratio $\left.t_{\|} / t_{\perp} \approx 4000[21]\right)$. Such a high anisotropy can raise the issue of incoherent electron motion in the perpendicular, $z$-direction. 22, 23 In the case of weakly incoherent interlayer motion, 23. the intralayer electron momentum is conserved in the tunneling process and the electron wave function in adjacent layers has some overlap, but there are many in-plane collisions between tunneling events. In this case as well as for coherent interlayer motion, the use of the quasiparticle spectrum Eq. 24 is legitimate. In the case of strongly incoherent interlayer transport, however, the intralayer electron momentum is not conserved in the tunneling processes between adjacent layers because the tunneling can be accompanied by strong elastic or inelastic processes. This case needs a separate treatment.

The tight-binding spectrum Eq. (24) for $a p_{F} \ll 1$ reduces to the form Eq. (16). In most cases considered here the use of the low-density spectrum Eq. (16) in the microscopic calculations is well justified.

\section{B. Electron-phonon vertex}

The electron-phonon part of the Hamiltonian, according to Walker, Smith, and Samokhin [9], is given by

$$
\begin{aligned}
& H_{e-p h}=-\sqrt{2} i \sum_{\mathbf{k}, \mathbf{p}, \mathbf{R}} G_{\mathbf{R}}\left(\frac{\hbar \omega_{0}(\mathbf{k})}{N M v_{s}^{2}}\right)^{1 / 2} \\
& \times(\widehat{\mathbf{k}} \cdot \widehat{\mathbf{R}})(\widehat{\mathbf{e}} \cdot \widehat{\mathbf{R}})(\cos \mathbf{p} \cdot \mathbf{R}) c_{\mathbf{p}+\mathbf{k}, \sigma}^{\dagger} c_{\mathbf{p}, \sigma}\left(a_{-\mathbf{k}}^{\dagger}+a_{\mathbf{k}}\right),
\end{aligned}
$$

where $\widehat{\mathbf{k}}$ is a unit vector in the direction of the phonon momentum, $\widehat{\mathbf{e}}$ a unit vector for the phonon polarization, $\mathbf{R}$ are the nearest bonds that are stretched by the sound wave. We have also defined $\omega_{0}(\mathbf{k})=v_{s} k$ the sound frequency, $v_{s}$ the sound velocity, $M$ the ion mass, $N$ the number of unit cells, $G_{\mathbf{R}}$ a constant that depends on the derivative of the hopping integral along the bond $\mathbf{R}$ with respect to the strain, and finally $a_{\mathbf{k}}^{(\dagger)}$ and $c_{\mathbf{p}, \sigma}^{(\dagger)}$ are, respectively, destruction and creation operators for phonons and for electrons of spin $\sigma$.

For longitudinal phonons propagating in the perpendicular direction, the main terms in the sum over $\mathbf{R}$ in Eq. (26) are those that contain the nearest neighbor interlayer bonds $\mathbf{R}= \pm \mathbf{c}$ (see Fig. 1(a)). For these bonds, the wave vector and the phonon polarization will satisfy $(\widehat{\mathbf{k}} \cdot \widehat{\mathbf{R}})(\widehat{\mathbf{e}} \cdot \widehat{\mathbf{R}})=1$. The electron-phonon vertex becomes

$$
\Gamma_{e p}^{(L)}(\mathbf{p})=g_{L} \cos \left(p_{z} c\right)
$$

where $g_{L}$ is a constant.

For transverse phonons, stretching of the nearest neighbor bonds Fig. 1(a) does not contribute to the sound attenuation as for these bonds $(\widehat{\mathbf{e}} \cdot \widehat{\mathbf{R}})=0$. In other words, the shear wave does not interact with electrons to this order. One should consider next-to-nearest neighbor bonds, that in our particular case are the diagonals of the sides of 3D $a \times a \times c$ unit cell. These bonds belong to the set $\{( \pm a, 0, \pm c),(0, \pm a, \pm c)\}$. Let us assume that the phonon polarization makes an angle $\varphi$ with the $x$-axis in the plane, such that $\widehat{\mathbf{e}}=\{\cos \varphi, \sin \varphi, 0\}$. The summation over the next-to-nearest neighbor bonds results in 


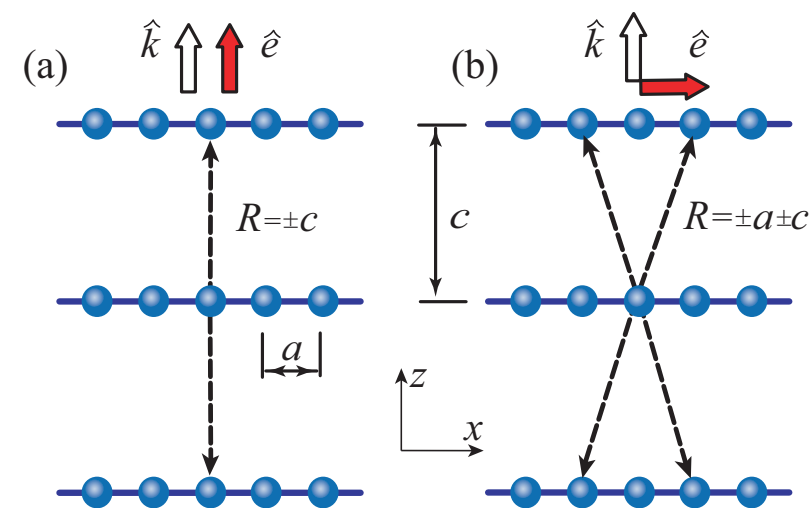

FIG. 1: (Color online) Side view of the basis vectors that determine the electron-phonon vertex for (a) longitudinal (L), and (b) transverse $(\mathrm{T})$ phonons propagating perpendicular to the layers. Here $a$ is the in-plane lattice period, $c$ the interlayer distance, and $\mathbf{R}$ are the nearest neighbor distances for the bonds that give the leading contribution to the electronphonon vertex, for both types of polarization.

an electron-phonon vertex of the form

$$
\Gamma_{e p}^{(T)}(\varphi, \mathbf{p})=g_{T}\left[\sin \left(p_{x} a\right) \cos \varphi+\sin \left(p_{y} a\right) \sin \varphi\right] \sin \left(p_{z} c\right),
$$

where $g_{T}$ involves the derivative of the transverse hopping $t_{X}$ with respect to strain. In the particular case of sound polarization parallel to the $x$-axis, the nearest neighbor bonds that give the leading contribution to the sound attenuation, are determined by: $\mathbf{R}= \pm \mathbf{a} \pm \mathbf{c}$ (see Fig. 1(b)). Summation over these bonds yields

$$
\Gamma_{e p}^{(T)}(\varphi=0, \mathbf{p})=g_{T} \sin \left(p_{x} a\right) \sin \left(p_{z} c\right) .
$$

In a similar manner, for phonon polarization oriented along $y$-axis, the vertex is

$$
\Gamma_{e p}^{(T)}(\varphi=\pi / 2, \mathbf{p})=g_{T} \sin \left(p_{y} a\right) \sin \left(p_{z} c\right) .
$$

It is natural to expect that the transverse coupling $g_{T}$ is significantly less than the longitudinal one $g_{L}$. Still, it can provide a sizable effect, according to preliminary experiments in organics that suggest that the mechanism of the sound attenuation has electronic origin at $T \rightarrow T_{c}$. At the same time, we neglect the contributions from all further neighbor bonds that lie for example along the spatial diagonals of the 3D unit cell, assuming that the magnitudes of corresponding hopping integrals decay fast enough.

Finally, note that the single-particle spectrum Eq. (24) should be modified because of the diagonal hopping $t_{X}$ mentioned above. That will introduce the term given by Eq. (58). However, we will see in the next section that this effect leads to small corrections, even in the Aslamazov-Larkin diagram, where it is potentially important, because $t_{X} / t_{\perp} \ll 1$.

\section{ULTRASOUND ATTENUATION AND SOUND VELOCITY}

\section{A. Normal state}

We start with the sound attenuation and sound velocity in the normal state. The sound attenuation coefficient is determined by the imaginary part $\gamma(k)$ of the complex frequency $\omega(k)$ where the pole of the phonon Green function $D(\mathbf{k}, \omega)$ is located. This quantity obeys Dyson's equation [24]

$$
D^{-1}\left(\mathbf{k}, \omega_{\nu}\right)=\left[D^{0}\left(\mathbf{k}, \omega_{\nu}\right)\right]^{-1}-\Pi\left(\mathbf{k}, \omega_{\nu}\right) .
$$

Expressed in bosonic Matsubara frequencies $\omega_{\nu}=2 \pi \nu T$ using units $k_{B}=1 \hbar=1$, with $\nu$ an integer, the quantity $D^{0}\left(\mathbf{k}, \omega_{\nu}\right)$

$$
D^{0}\left(\mathbf{k}, \omega_{\nu}\right)=-\frac{\omega_{0}^{2}(\mathbf{k})}{\omega_{\nu}^{2}+\omega_{0}^{2}(\mathbf{k})}
$$

is the phonon propagator in the non-interacting case, and $\Pi\left(\mathbf{k}, \omega_{\nu}\right)$ is the phonon self-energy:

$$
\begin{aligned}
& \Pi\left(k, \omega_{\nu}\right) \\
= & 2 T \sum_{\varepsilon_{n}} \int \frac{d^{3} p}{(2 \pi)^{3}} \Gamma_{e p}^{2} G\left(\varepsilon_{n}, p\right) G\left(\varepsilon_{n}+\omega_{\nu}, p+k\right) .
\end{aligned}
$$

In Eq. (32) the electron Green function $G\left(\mathbf{p}, \varepsilon_{n}\right)$ at finite temperature in the presence of impurities, is given by

$$
G\left(\mathbf{p}, \varepsilon_{n}\right)=\frac{1}{i \tilde{\varepsilon}_{n}-\xi(\mathbf{p})},
$$

where $\xi(\mathbf{p})$ is the quasiparticle energy and where we defined $\tilde{\varepsilon}_{n}=\varepsilon_{n}+1 /(2 \tau) \operatorname{sign}\left(\varepsilon_{n}\right)$ with $\varepsilon_{n}=\pi T(2 n+1)$ the Matsubara frequency and $\tau$ the electronic elastic scattering time. The quantity $i /(2 \tau) \operatorname{sign}\left(\varepsilon_{n}\right)$ is the imaginary part of the quasiparticle self-energy. The real part of the self-energy is constant and is absorbed in the definition of the chemical potential. 24]

The scattering time $\tau$ in Eq. (32) is the only effect of impurity averaging in the polarization operator. We do not include the corrections to the electron-phonon vertices $\Gamma_{e p}$. In the case of the current-current correlator, vertex corrections lead to the replacement of the scattering time $\tau$ by its transport analog $\tau_{t r}$. 24] For $s$-wave scattering, vertex corrections vanish because the vector vertex $e v_{\alpha}$ averages to zero upon angular integration at vanishing external momentum k. For stress tensor correlator, that we need in tight-binding limit, one can use results of Schmid [6]. He showed that in the continuum limit, taking into account perfect screening, there is no impurity diffusion enhancement of the electron-phonon vertex in the case of transverse phonons, and that for longitudinal phonons this effect is negligible in the hydrodynamic limit $k \ell \ll 1$ and $\omega \tau \ll 1$. Physically, this comes about from the fact that the calculation should be 
done in the moving frame and that screening is perfect at long wavelengths. In our case, the analogous argument of electroneutrality leads [9] to the replacement of the stress vertex $F$ by $F-\langle F\rangle$, where the average accounts for the chemical potential shift. 25] That average is precisely what is needed to make the impurity vertex correction vanish. In addition, in our specific case, $\langle F\rangle=0$ to the order in phonon wave vector $k$ that we need.

As usual, after integration over $p$ and summation over $\varepsilon_{n}$ in Eq. (32), one should make the analytic continuation of the external phonon frequency following the rule $i \omega_{\nu} \rightarrow \omega+i \delta$. For sound propagating along $z$-axis, in the hydrodynamic limit $\omega \tau \ll 1$, it suffices to set $k=0$ and expand the integrals in powers of $\omega \tau$. The subtleties and the typical calculation details are give in Ref. 15], to which we will refer in what follows.

The power attenuation can be obtained from

$$
\alpha(\omega)=-2 \gamma(\omega) / v_{s}
$$

where

$$
\gamma(\omega)=\frac{1}{2} \omega_{0}(k) \operatorname{Im}\left[\Pi^{R}(\omega)\right],
$$

and $v_{s}$ is the sound velocity.

The renormalization of the phonon frequency $\omega(k)$ is obtained from the real part of phonon self-energy using

$$
\omega(k)=\omega_{0}(k) \sqrt{1+\operatorname{Re} \Pi^{R}} .
$$

For both longitudinal and transverse sound polarizations, one finds:

$$
\begin{aligned}
& \alpha(\omega, \widehat{\mathbf{e}})=\frac{g_{\hat{e}}^{2} \nu_{0} \omega^{2} \tau}{v_{s}}, \\
& v_{s}(\omega, \widehat{\mathbf{e}})=-g_{\hat{e}}^{2} \nu_{0},
\end{aligned}
$$

where index $\hat{e}$ denotes the phonon polarization ( $\mathrm{T}$ or $\mathrm{L}$ ). One can see that for transverse polarization, the phonon self-energy does not depend on the orientation of the vector $\widehat{\mathbf{e}}$ in the plane. This is true for both tight-binding form of the spectrum Eq. (24) and low-density limit Eq. (16). In the latter case, one can analytically obtain:

$$
\int_{0}^{2 \pi}\left(\Gamma_{e p}^{(T)}\right)^{2} d \varphi_{p} \sim \int_{0}^{2 \pi} \cos ^{2}\left(\varphi-\varphi_{p}\right) d \varphi_{p}
$$

that does not depend on $\varphi$.

\section{B. Sound attenuation at $T<T_{c}$}

Before analyzing the fluctuation effects, let us consider the effect of the in-plane phonon polarization and order parameter symmetry on sound attenuation at temperature quite below $T_{c}$. It is possible to show [9] that the sound attenuation in the Born limit is given by:

$$
\frac{\alpha(T)}{\alpha\left(T_{c}\right)}=\int_{0}^{\infty} d E\left(-\frac{\partial f}{\partial E}\right) \frac{A(E)}{E}
$$

where

$$
A(E)=\frac{\left\langle\Gamma_{e p}^{2}(\varphi) \operatorname{Re} \sqrt{E^{2}-\left|\Delta_{p}\right|^{2}}\right\rangle_{F . S .}}{\left\langle\Gamma_{e p}^{2}(\varphi)\right\rangle_{F . S .}},
$$

with $\Delta_{p}$ the superconducting energy gap, $E=$ $\sqrt{\xi^{2}+\left|\Delta_{p}\right|^{2}}$, and $f(E)$ the Fermi distribution function. For longitudinal phonons propagating in $s$ - and $d$-wave superconductor, $\Gamma_{e p}$ does not depend on $\varphi$. For transverse phonons in $s$-wave superconductor the attenuation does not depend on the polarization direction because the energy gap $\Delta_{p}$ is isotropic in the plane, and because of Eq. (38). Of course, the dependence of the attenuation on temperature can be different for different order parameter symmetry. [9, 10, 11]

Let us consider the fourth case, namely, transverse phonons in $d$-wave superconductor, at the energy gap given by $\Delta_{p}=\Delta_{0} \cos \left(2 \varphi_{p}\right)$. Let phonons propagate perpendicular to the conduction layers (along the $\hat{z}$ axis, Fig. 21), with the angle $\varphi$ between the phonon polarization vector $\widehat{\mathbf{e}}$ and the $x$ axis in plane. The electron-phonon

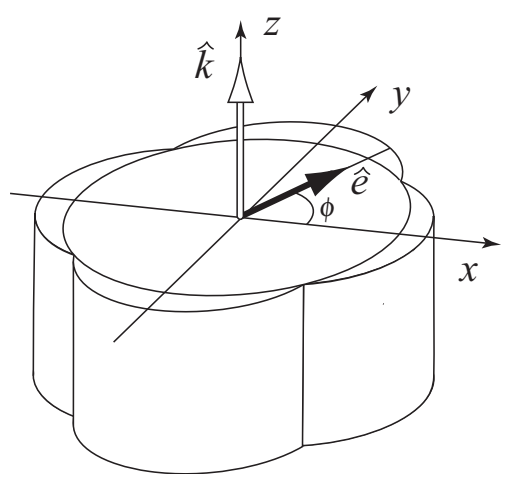

FIG. 2: Sketch showing the $d$-wave superconducting energy gap. The ultrasound is propagating along $\hat{\mathbf{k}}$ direction with the polarization $\widehat{\mathbf{e}}$ having an angle $\varphi$ with the $x$ axis.

vertex is then given by Eq. (27). As was pointed out in Sec. IVA in the normal state the sound attenuation and sound velocity do not depend on the orientation of the polarization in plane. Combining the expressions Eqs. [27), 391, and (40), one can see that the sound attenuation does not depend on the polarization direction below $T_{c}$ as well. This can be demonstrated analytically in lowdensity limit using Eq. (38), and numerically in more general case of a square quasi-2D lattice. This should be contrasted with the results of Refs. [9] and [10], where the attenuation of transverse phonons with both $\widehat{\mathbf{k}}$ and $\widehat{\mathbf{e}}$ lying in the plane does depend on the propagation direction. 


\section{FLUCTUATION CORRECTIONS, S-WAVE SUPERCONDUCTOR}

\section{A. Generalities}

The Feynman diagrams that give the main contribution to the renormalization of the electron-phonon loop Eq. (32) by superconducting fluctuations are presented in Fig. 3. Here, each wavy line corresponds to the fluc-
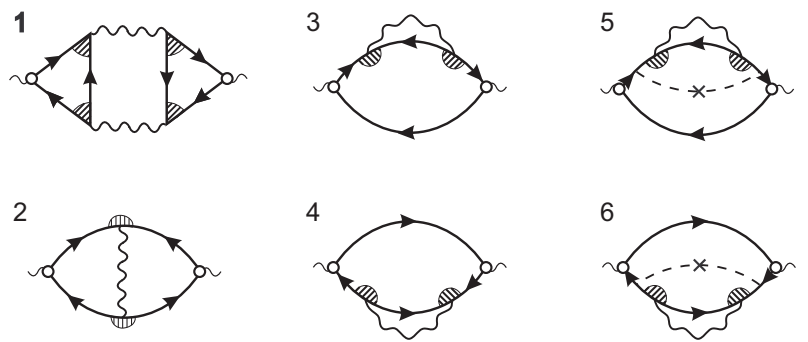

FIG. 3: Feynman diagrams that give the leading-order corrections from the superconducting $s$-wave fluctuations to the sound attenuation as $T \rightarrow T_{c}$. Diagram 1 is of the AslamazovLarkin (AL) type, diagram 2 is of Maki-Thompson (MT) type, and the diagrams 3-6 are of the density of states (DOS) type. Solid lines are the normal state Green functions, wavy lines the fluctuation propagators, shaded semicircles the impurity ladder averaging, dashed lines with cross the single impurity scattering, and open circles the renormalized electron-phonon vertices. The corresponding diagrams with a $d$-wave order parameter symmetry do not contain impurity semicircles (See Ref. [27]).

tuation propagator (Cooper ladder) $L\left(q, \Omega_{k}\right)$ which, as $T \rightarrow T_{c}$, has the form [19]:

$L^{(s)}\left(q, \Omega_{k}\right)^{-1}=-\nu_{0}\left[\epsilon+\eta q_{\|}^{2}+r \sin ^{2}\left(q_{z} c / 2\right)+\Omega_{k} \tau_{s}\right]$,

where $\epsilon \approx\left(T-T_{c}\right) / T_{c}, \Omega_{k}$ is the bosonic Matsubara frequency, and the coefficient $\eta$ has the meaning of the square of the effective coherence length $\xi$ in the isotropic $2 \mathrm{D}$ case for $s$-wave pairing: [19]

$$
\begin{aligned}
& \eta \equiv \xi^{2}(T \tau)=-\frac{\left(\tau v_{F}\right)^{2}}{2} \\
& \times\left.\left[\psi\left(\frac{1}{2}+\frac{1}{4 \pi x}\right)-\psi\left(\frac{1}{2}\right)-\frac{1}{4 \pi x} \psi^{\prime}\left(\frac{1}{2}\right)\right]\right|_{x=T \tau}
\end{aligned}
$$

where $\psi(z)$ is the digamma function. Then, $r=$ $16 t_{\perp}^{2} \eta / v_{F}^{2} \ll 1$ is the anisotropy parameter [26]. At $T=T_{c}$, the anisotropy parameter can be written as $r=4 \xi_{\perp}^{2}(0) / c^{2}$ where $\xi_{\perp}(0)$ is the Cooper pair size in the perpendicular $(z)$ direction. Finally, $\tau_{s}=\pi /(8 T)$ is the Ginzburg-Landau time.

Returning to Fig. 3] the shaded semicircles correspond to vertex corrections from impurity averaging and are given by:

$$
\lambda\left(\mathbf{q}, \varepsilon_{1}, \varepsilon_{2}\right)=\frac{\left|\tilde{\varepsilon}_{1}-\tilde{\varepsilon}_{2}\right|}{\left|\varepsilon_{1}-\varepsilon_{2}\right|+\frac{\widehat{D} q^{2}}{\tau^{2}\left|\tilde{\varepsilon}_{1}-\tilde{\varepsilon}_{2}\right|^{2}} \Theta\left(-\varepsilon_{1} \varepsilon_{2}\right)} .
$$

We neglect the contribution from the diagrams that contain the impurity ladder in the particle-particle channel 15] because they are less singular in $\epsilon$ (they are not shown on Fig. [3).

The open circles at the extreme left and right-hand sides of the diagrams in Fig. 3 represent the electronphonon vertices $\Gamma_{e p}(\mathbf{p})$, that contain the dependence on electron momentum and that will be different for longitudinal and transverse phonon polarizations. Note that there is no impurity averaging of the vertices in fluctuation diagrams just like in the normal state polarization operator in the hydrodynamic limit. [6] .

As usual, the diagrams in Fig. 3 correspond to three different manifestations of superconducting fluctuations. [19] a) Some of the electrons behave like Cooper pairs for a time given by the Ginzburg-Landau time. This is the famous Aslamazov-Larkin (AL) contribution Fig. 3](1). b) The single-particle excitations are Andreev reflected off the superconducting fluctuations, as described by the so-called Maki-Thompson (MT) term Fig. 3(2). c) The effective number of normal carriers is reduced because some of the electrons exist as transient Cooper pairs. This is the so-called density of states (DOS) contribution in Fig. 3 (3-6). Additionally, the analytical expression for the MT term can be separated into the anomalous (aMT) and regular (rMT) parts. The difference between them comes from the fact that there is additional diffusion pole in the integral for the anomalous MT part, that in most cases enhances the aMT fluctuation corrections. 19]

The evaluation of the integrals corresponding to the diagrams in Fig. 3 includes taking the limit $\Omega_{k}=0$ in the Green's functions and also in the fluctuation propagator of the DOS and MT diagrams, and analytically continuing the diagrams to real phonon frequencies $\omega_{\nu} \rightarrow-i \omega$. In the hydrodynamic limit, where the electron mean free path $\ell$ is much smaller than the wavelength of sound and where the electron collision rate $\tau^{-1}$ is much larger than the sound frequency, we expand the result in powers of $\omega \tau$ and we set the phonon momentum $k=0$ because the expansion in powers of $k \ell$ gives corrections negligible to leading order of $\omega \tau$. The terms with odd powers of $\omega$ contribute to the sound attenuation while the terms proportional to even powers of $\omega$ contribute to the sound velocity. In the most general form the corrections can be written as:

$$
\begin{gathered}
\Delta \alpha^{(\beta, \hat{e}, s)}(T, \omega)=\frac{g_{\hat{e}}^{2} \omega^{2} \nu_{0}}{\varepsilon_{F} v_{s}} \kappa_{\alpha}^{(\beta, \hat{e}, s)}(T \tau) f_{\alpha}^{(\beta, s)}\left(\epsilon, r, \gamma_{\phi}\right), \\
\frac{\Delta \omega^{(\beta, \hat{e}, s)}(T, \omega)}{\omega}=\frac{\Delta v_{s}^{(\beta, \hat{e}, s)}(T, \omega)}{v_{s}} \\
=\frac{g_{\hat{e}}^{2} T \nu_{0}}{\varepsilon_{F}} \kappa_{v}^{(\beta, \hat{e}, s)}(T \tau) f_{v}^{(\beta, s)}\left(\epsilon, r, \gamma_{\phi}\right) .
\end{gathered}
$$

Here $\beta$ denotes the particular channel (DOS, rMT, aMT or $\mathrm{AL}$ ), superscript $\hat{e}$ the phonon polarization (L or $\mathrm{T}$ ). The superscript $s$ stands for the $s$-wave symmetry of the order parameter and in following Sections on $d$-wave 
symmetry it can be also $d$. The symbol $f^{(\beta, s)}\left(\epsilon, r, \gamma_{\phi}\right)$ denotes the function of temperature which usually contains the main singularity and comes from the integration over the momentum $\mathbf{q}$ in the fluctuation propagator Eq. (41), and which also depends on the material properties. The $\gamma_{\phi}=2 \eta /\left(v_{F}^{2} \tau \tau_{\phi}\right)$ in the temperature functions is the cutoff parameter that appears in the anomalous MT integrals and that depends on the phase-breaking time $\tau_{\phi}$. 14] When the subscripts $\alpha$ or $v$ on $f^{(\beta, s)}\left(\epsilon, r, \gamma_{\phi}\right)$ are omitted it is because the function is identical for the attenuation and sound velocity cases.

\section{B. Longitudinal phonons}

For $s$-wave longitudinal case, the analytical expressions for the temperature functions $f^{(\beta, s)}$ read: 15.

$$
\begin{aligned}
& f^{(D O S, s)}(\epsilon, r)=\ln \left(\frac{2}{\sqrt{\epsilon}+\sqrt{\epsilon+r}}\right), \\
& f^{(r M T, s)}(\epsilon, r)=\frac{(\sqrt{\epsilon}-\sqrt{\epsilon+r})^{2}}{r}, \\
& f^{(a M T, s)}\left(\epsilon, r, \gamma_{\phi}\right) \\
& =\frac{1}{r}\left[\frac{\epsilon+r+\gamma_{\phi}}{\sqrt{\gamma_{\phi}\left(\gamma_{\phi}+r\right)}+\sqrt{\epsilon(\epsilon+r)}}-1\right], \\
& f_{\alpha}^{(A L, s)}(\epsilon, r)=\frac{1}{r}\left[1-\sqrt{\frac{\epsilon}{\epsilon+r}}\left(1+\frac{r}{2(\epsilon+r)}\right)\right], \\
& f_{v}^{(A L, s)}(\epsilon, r)=\left[1+\frac{2 \epsilon}{r}\left(-1+\sqrt{\frac{\epsilon}{\epsilon+r}}\right)\right] .
\end{aligned}
$$

The coefficients $\kappa_{\alpha}^{(\beta, \hat{e}, s)}(T \tau)$ in Eq. (44) and $\kappa_{v}^{(\beta, \hat{e}, s)}(T \tau)$ in Eq. (45) come from the integration of Green functions and impurity blocks. They have weaker temperature dependence as $\epsilon \rightarrow 0$ than $f^{(\beta, s)}\left(\epsilon, r, \gamma_{\phi}\right)$, and they basically show how the impurity concentration affects the result. In Eqs. (49) and (50) we introduced indices $\alpha$ and $v$ to distinguish between the temperature corrections to the sound attenuation and the sound velocity that are different in the AL channel.

The analytical form and an extended discussion of the asymptotics of the $f^{(\beta, s)}\left(\epsilon, r, \gamma_{\phi}\right)$ functions and the $\kappa$ coefficients can be found in Ref. [15]. In short, the temperature functions experience sharp enhancement as $\epsilon \rightarrow 0$, although they remain finite at $\epsilon=0$. Note that $f^{(r M T, s)}(\epsilon=0, r)=f_{v}^{(A L, s)}(\epsilon=0, r)=1$, while the value of functions $f^{(D O S, s)}(\epsilon, r), f^{(a M T, s)}\left(\epsilon, r, \gamma_{\phi}\right)$ and $f_{\alpha}^{(A L, s)}(\epsilon, r)$ can be much larger than 1 at high material anisotropy. In addition, long phase breaking times increase $f^{(a M T, s)}\left(\epsilon, r, \gamma_{\phi}\right)$. Thus, with the parameters corresponding to the real materials, the rMT diagram is always negligible. In contrast to the conductivity fluctuations, the contributions of the DOS diagram is enhanced and becomes comparable to that of the AL and aMT diagrams, although its sign is opposite to that of AL. The superconducting fluctuation corrections to the sound at- tenuation, in a realistic range of parameters, are given by the sum of the DOS, anomalous MT and AL terms, which decrease the normal state attenuation. In contrast, to leading order in $\omega$, the corrections to the sound velocity are given by DOS diagrams, because the expansion for the anomalous MT diagram begins at order $\omega$ while the AL diagram is small to this order.

One can also see that the temperature dependence of the microscopic terms Eqs. (46), (47), and (50) is in agreement with the thermodynamic results Eqs. (19)(21) that depend on the modulation of the hopping integral.

Summarizing, the signs of the principal terms for $s$ wave longitudinal case is as follows:

$$
\begin{array}{ll}
\Delta \alpha^{(D O S, L, s)}<0, & \Delta \alpha^{(a M T, L, s)}<0, \\
\Delta v^{(D O S, L, s)}>0, & \Delta \alpha^{(A L, L, s)}>0,
\end{array}
$$

and all other terms can be neglected.

\section{Transverse phonons}

In the $s$-wave transverse case, the structure of the DOS and MT terms is as follows:

$$
\begin{aligned}
& \Pi^{(\beta, T, s)}\left(\omega_{\nu}\right) \\
& =2 g_{T}^{2} T \sum_{\Omega_{k}} \int \frac{d^{3} q}{(2 \pi)^{3}} L^{(s)}\left(q, \Omega_{k}\right) K^{(\beta, T, s)}\left(q, \Omega_{k}, \omega_{\nu}\right),
\end{aligned}
$$

where $K^{(\beta, T, s)}\left(q, \Omega_{k}, \omega_{\nu}\right)$ is the "bubble" that contains electronic Green functions Eq. (33), impurity vertices Eq. (43), and electron-phonon vertices Eq. (27). There is no significant modification of the integrals in comparison with the longitudinal polarization case. Indeed, the angular dependence of the electron-phonon vertex part $\left[\Gamma_{e p}^{(T)}(\varphi, \mathbf{p})\right]^{2}$ in the DOS diagram is averaged out at the Fermi-surface.

In the Maki-Thompson diagram, the term $\Gamma_{e p}^{(T)}(\varphi, \mathbf{p}) \Gamma_{e p}^{(T)}(\varphi, \mathbf{q}-\mathbf{p})$ after averaging over the direction of $\mathbf{p}$ provides an additional term $\cos \left(q_{x} a\right)$ in the integrals over $\mathbf{q}$ :

$$
\int \frac{d^{3} q}{(2 \pi)^{3}} \frac{\cos \left(q_{x} a\right) \cos \left(q_{z} c\right)}{\epsilon+\eta q_{\|}^{2}+r \sin ^{2}\left(q_{z} c / 2\right)},
$$

for the regular part, and

$$
\int \frac{d^{3} q}{(2 \pi)^{3}} \frac{\cos \left(q_{x} a\right) \cos \left(q_{z} c\right)}{\left(\hat{D} q^{2}+1 / \tau_{\phi}\right)\left[\epsilon+\eta q_{\|}^{2}+r \sin ^{2}\left(q_{z} c / 2\right)\right]} .
$$

for the anomalous part of the diagram. (We set $\varphi=0$ from now on, assuming the polarization is along the $x$ axis. The generalization of our results to arbitrary polarization is straightforward and does not change our main conclusions). At $a p_{F} \ll 1$, one can neglect the 
$q_{x}$-dependence in $\cos \left(q_{x} a\right)$ in the above integrals and immediately obtain the longitudinal MT terms Eqs. (47) and (49), with the properly defined transverse coupling constant $g_{T}$.

The Aslamazov-Larkin diagram is given by:

$$
\begin{gathered}
\Pi^{(A L)}\left(\omega_{\nu}\right)=-2 g_{T}^{2} T \sum_{\Omega_{k}} \int \frac{d^{3} q}{(2 \pi)^{3}} B^{2}\left(q, \Omega_{k}, \omega_{\nu}\right) \\
\times L^{(s)}\left(q, \Omega_{k}\right) L^{(s)}\left(q, \Omega_{k}+\omega_{\nu}\right)
\end{gathered}
$$

where $B\left(q, \Omega_{k}, \omega_{\nu}\right)$ is the triangular block in the diagram (1) on Fig. 3 As usual, at $q \rightarrow 0$, which gives the main contribution from fluctuation propagators, the Green function $G(q-p)$ in the B-block should be expanded in powers of $q$ :

$$
\begin{aligned}
G(q-p) & =G(-p)+G^{2}(-p) \Delta \xi(q, p) \\
& +G^{3}(-p) \Delta \xi(q, p)^{2}+\ldots
\end{aligned}
$$

where $\Delta \xi(q, p)=\xi(q-p)-\xi(p)$. One can see that with the quasiparticle spectrum Eq. (16) one obtains

$$
\begin{aligned}
\Delta \xi(q, p)= & \frac{q_{x}\left(q_{x}-2 p_{x}\right)}{2 m} \\
& +2 t_{\perp}\left\{\cos \left[\left(q_{z}-p_{z}\right) c\right]-\cos \left(p_{z} c\right)\right\} .
\end{aligned}
$$

The first term of the expansion Eq. (56) that does not vanish upon angular averaging is $G(-p)^{3}(\Delta \xi(q, p))^{2}$. Thus the B-block contains a product of five Green functions; one can check that this does not lead to any singularity in the total AL diagram contribution.

We must also consider the term in the quasiparticle energy spectrum that depends on hopping $t_{X} \ll t_{\perp}$ along the diagonal lattice bonds $\mathbf{R}= \pm \mathbf{a} \pm \mathbf{c}$ (See Fig. (1). This term can be chosen as:

$$
\xi(p)_{X}=t_{X} \sin \left(p_{x} a\right) \sin \left(p_{z} c\right),
$$

such that

$$
\begin{aligned}
& \Delta \xi(q, p)_{X}=\frac{q_{x}\left(q_{x}-2 p_{x}\right)}{2 m} \\
& +t_{X}\left\{\sin \left[\left(q_{x}-p_{x}\right) a\right] \sin \left[\left(q_{z}-p_{z}\right) c\right]-\sin \left(p_{x} a\right) \sin \left(p_{z} c\right)\right\} .
\end{aligned}
$$

With this modification, the first non-vanishing term of the expansion Eq. (561) appears to first order in $\Delta \xi(q, p)_{X}$. The integration in the B-block now is reduced to $\int\left\langle\Gamma_{e p} \Delta \xi\right\rangle_{F . S .} G(p) G(p+k) G^{2}(-p) d \xi_{p}$; one can see that the integration over $d \xi_{p}$ gives the same result both for longitudinal and transverse polarizations. Indeed, there is no angular dependence in the integral $\int G(p) G(p+$ $k) G^{2}(-p) d \xi_{p}$. Then, the average $\left\langle\Gamma_{e p}^{(T)} \Delta \xi_{X}\right\rangle_{F . S \text {. contains }}$ a factor:

$$
g_{T} t_{X} \frac{\pi^{2}}{c}\left[1-\cos \left(a q_{x}\right) \cos \left(c q_{z}\right)\right]
$$

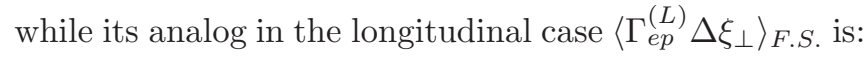

$$
g_{L} t_{\perp} \frac{\pi^{2}}{c}\left[1-\cos \left(c q_{z}\right)\right]
$$

Integrating over $q$ in Eq. (55) with Eq. (60) one can set $\cos \left(a q_{x}\right) \approx 1$, thus reducing it to Eq. (661). From Eq. (26) of Ref. 15], one can easily obtain the expression for the B-block:

$$
B(q)=-\frac{t_{X} \eta \nu_{0}\left[1-\cos \left(c q_{z}\right)\right]}{v_{F}^{2}} .
$$

We see that the difference between the longitudinal and transverse sound attenuation and velocity in AL diagram is only in the prefactor. However, compared with the DOS and MT contributions, this prefactor contains an additional $\left(t_{X} / t_{\perp}\right)^{2}$ term because of the anisotropy parameter $r$ which enters the fluctuation propagator which is still proportional to $t_{\perp}^{2}$. One would expect that this prefactor satisfies $\left(t_{X} / t_{\perp}\right)^{2} \ll 1$, thus making the AL term negligible.

In summary, the main contribution to the fluctuation corrections is given by the DOS diagrams (and aMT diagrams in sound attenuation at sufficiently long pairbreaking times). The temperature function of the rMT diagram is less singular and the AL diagram contains an additional small factor, thus being negligible. The signs of the principal terms are as follows:

$$
\begin{aligned}
& \Delta \alpha^{(D O S, T, s)}<0, \quad \Delta \alpha^{(a M T, T, s)}<0, \\
& \Delta v^{(D O S, T, s)}>0 .
\end{aligned}
$$

\section{FLUCTUATION CORRECTIONS, D-WAVE}

\section{A. Modification of the $d$-wave integrals}

If we assume that the virtual Cooper pairs have $d$ wave symmetry, the modification of the calculations is as follows.

We have to take into account the momentum dependence of a pairing interaction that now can be written as:

$$
V\left(p, p^{\prime}\right)=\eta\left(\varphi_{p}\right) g \eta\left(\varphi_{p^{\prime}}\right),
$$

where, for $d$-wave pairing, $\eta\left(\varphi_{p}\right) \sim \cos \left(a p_{x}\right)-\cos \left(a p_{y}\right) \propto$ $\sqrt{2} \cos \left(2 \varphi_{p}\right)$.

Then, in all fluctuation diagrams of Fig. 3] each wavy line corresponds to the fluctuation propagator with additional symmetry factors:

$$
\eta\left(\varphi_{p}\right) L^{(d)}\left(q, \Omega_{k}\right) \eta\left(\varphi_{p^{\prime}}\right),
$$

where $L^{(d)}\left(q, \Omega_{k}\right)$ has the same form as the $s$-wave fluctuation propagator Eq. (41) with modified $\epsilon, \xi, \tau$, and $r$ :

$$
L_{d}(q, \Omega)^{-1}=-\nu_{0}\left[\epsilon_{d}+\eta_{d} q_{\|}^{2}+r_{d} \sin ^{2}\left(c q_{z} / 2\right)-i \Omega \tau_{d}\right] .
$$

In the quasi-2D case, and assuming that the phasebreaking processes contribute only as the upper cutoff 
for the Maki-Thompson diagram, the coefficients in Eq. (66) read (compare with those of Ref. [28]):

$$
\begin{aligned}
& \epsilon_{d}=\ln \frac{T}{T_{c}}-\psi\left(\frac{1}{2}+\frac{1}{4 \pi T_{c} \tau}\right)+\psi\left(\frac{1}{2}+\frac{1}{4 \pi T \tau}\right) \\
& \approx \epsilon\left[1-\frac{1}{4 \pi x} \psi^{\prime}\left(\frac{1}{2}+\frac{1}{4 \pi x}\right)\right] \\
& \approx \epsilon \begin{cases}13.1595 x^{2}, & x \ll 1, \\
1, & x \gg 1 ;\end{cases} \\
& \eta_{d}=\left(\frac{v_{F} \tau}{8 \pi x}\right)^{2}\left|\psi^{\prime \prime}\left(\frac{1}{2}+\frac{1}{4 \pi x}\right)\right| \\
& \approx\left(\frac{v_{F}}{2 T}\right)^{2} \begin{cases}x^{2}, & x \ll 1, \\
\frac{7 \zeta(3)}{8 \pi^{2}}, & x \gg 1 ;\end{cases} \\
& \tau_{d}=\tau \frac{1}{4 \pi x} \psi^{\prime}\left(\frac{1}{2}+\frac{1}{4 \pi x}\right) \\
& \approx \frac{1}{T} \begin{cases}x, & x \ll 1, \\
\frac{\pi}{8}, & x \gg 1 ;\end{cases} \\
& r_{d}=\left(\frac{t_{\perp}}{4 \pi T}\right)^{2}\left|\psi^{\prime \prime}\left(\frac{1}{2}+\frac{1}{4 \pi x}\right)\right| \\
& \approx \frac{t_{\perp}^{2}}{T^{2}} \begin{cases}x^{2}, & x \ll 1, \\
\frac{7 \zeta(3)}{8 \pi^{2}}, & x \gg 1 .\end{cases}
\end{aligned}
$$

where $x=T \tau$. Note that the dependence of $\eta_{d}$ and $r_{d}$ on the impurity concentration (through the parameter $T \tau)$ is the same. It also should be noted that the $d$-wave parameters $\epsilon_{d}, \eta_{d}, r_{d}$, and $\tau_{d}$ coincide with the corresponding $s$-wave terms in the clean limit $(T \tau \gg 1)$. In the opposite, dirty limit, the pair-breaking effect of impurities for $d$-wave pairing makes these coefficient different from those for $s$-wave pairing.

Note also that in the expression Eq. (67) $T_{c}$ is the real superconducting transition temperature. In the case of $d$-wave pairing, it is renormalized even by scattering on non-magnetic impurities, and it satisfies the AbrikosovGorkov equation:

$$
\ln \frac{T_{c}}{T_{c 0}}-\psi\left(\frac{1}{2}\right)+\psi\left(\frac{1}{2}+\frac{1}{4 \pi \tau T_{c}}\right)=0 .
$$

In the clean case, at $\tau T \approx \tau T_{c} \gg 1$, there is not much difference between $T_{c}$ and $T_{c 0}$. In the opposite, dirty limit, when the elastic scattering time is small, $d$-wave superconductivity is suppressed and $T_{c} / T_{c 0} \rightarrow 0$ as $\tau T \rightarrow$ 0 . On the other hand, there is no such impurity effect on the $s$-wave $T_{c}$.

Finally, there is no impurity averaging in the fluctuation propagator (no shaded semicircles on diagrams Fig. 31. 27 This also leads to the absence of an anomalous part in the MT diagram 2 of Fig. 3 since there is no diffusion pole in the corresponding integral.

\section{B. Longitudinal phonons}

\section{DOS}

The corrections to the sound attenuation and to the sound velocity have the structure of Eqs. (44) and (45), with the temperature functions appropriate for $d$-wave pairing:

$$
\begin{array}{r}
f^{(D O S, d)}\left(\epsilon_{d}, r_{d}\right)=\ln \left(\frac{2}{\sqrt{\epsilon_{d}}+\sqrt{\epsilon_{d}+r_{d}}}\right) \\
\approx-\frac{1}{2}\left\{\begin{array}{cc}
\ln r_{d}, & \epsilon_{d} \ll r_{d}, \\
\ln \epsilon_{d}, & r_{d} \ll \epsilon_{d},
\end{array}\right.
\end{array}
$$

with $\epsilon_{d}$ and $r_{d}$ given by Eqs. (67) and (70). The renormalization of $\tau_{d}$ Eq. (69) does not affect the results because the main contribution from the fluctuation propagator is at $\Omega \rightarrow 0$.

The $\kappa^{(D O S, L, d)}$ coefficients are inversely proportional to the renormalized $\eta_{d}=\xi_{d}^{2}$ in Eq. (68) which is different from its $s$-wave analog. Another source of difference between $s$-wave and $d$-wave results is the absence of impurity vertices $\lambda$ in $d$-wave diagrams. With all these modifications, one finally obtains the dependence of the $\kappa^{(D O S, L, d)}$ coefficients on sample cleanness through the parameter $T \tau \approx T_{c} \tau$.

For sound attenuation, the coefficient is given by:

$$
\begin{gathered}
\kappa_{\alpha}^{(D O S, L, d)}(x)=\frac{1}{\pi \psi^{\prime \prime}\left(\frac{1}{2}+\frac{1}{4 \pi x}\right)} \\
\times\left[32 \pi^{2} x^{2} \psi^{\prime}\left(\frac{1}{2}+\frac{1}{4 \pi x}\right)+8 \pi(2 \pi-1) x \psi^{\prime \prime}\left(\frac{1}{2}+\frac{1}{4 \pi x}\right)\right. \\
\left.\quad+\psi^{\prime \prime \prime}\left(\frac{1}{2}+\frac{1}{4 \pi x}\right)\right] \\
\approx \begin{cases}4(2 \pi-3) x, & x \ll 1, \\
-\frac{4 \pi^{3} x^{2}}{7 \zeta(3)}, & x \gg 1,\end{cases}
\end{gathered}
$$

while for sound velocity it reads:

$$
\kappa_{v}^{(D O S, L, d)}=1 .
$$

The temperature function $f^{(D O S, d)}\left(\epsilon_{d}, r_{d}\right)$ Eq. (71) is similar to its $s$-wave analog Eq. [46], although now it also depends on the impurity concentration. Qualitatively, it is monotonically increasing as $T \rightarrow T_{c}$ just like $f^{(D O S, s)}(\epsilon, r)$.

\section{2. $r M T$}

Modifications similar to those in the DOS integrals also occur in the MT diagram. There is no aMT contribution because there is no diffusion pole originating from the impurity vertices. For rMT term, the temperature function 
is:

$$
\begin{gathered}
f^{(r M T, d)}\left(\epsilon_{d}, r_{d}\right)=\frac{\left(\sqrt{\epsilon_{d}}-\sqrt{\epsilon_{d}+r_{d}}\right)^{2}}{r_{d}} \\
\approx\left\{\begin{array}{cc}
1, & \epsilon_{d} \ll r_{d}, \\
\frac{r_{d}}{4 \epsilon_{d}}, & r_{d} \ll \epsilon_{d} .
\end{array}\right.
\end{gathered}
$$

Similarly to the $s$-wave case, the function in Eq. (75) has a limit $f^{(r M T, d)}\left(\epsilon_{d}=0, r_{d}\right)=1$. This function is very little affected by $T \tau$. The $\kappa^{(r M T, L, d)}$ coefficients are finite (they do not contain a singularity as functions of $x$ ). In summary, the rMT contributions to the attenuation and sound velocity is negligible in comparison with those from the DOS diagrams.

\section{3. $A L$}

The triangle of Green functions in diagram 1 in Fig. 3 does not contain impurity semicircles and reads:

$$
\begin{aligned}
& B_{d}^{(L)}\left(q, \Omega_{k}, \omega_{\nu}\right)=T \sum_{\varepsilon_{n}} \int \frac{d^{3} p}{(2 \pi)^{3}} \eta^{2}\left(\varphi_{p}\right) \Gamma_{e p}^{(L)}\left(p_{z}\right) \\
& \times G\left(p, \varepsilon_{n}\right) G\left(p, \varepsilon_{n}+\omega_{\nu}\right) G\left(q-p,-\varepsilon_{n}\right) .
\end{aligned}
$$

One can see that just like in the "longitudinal $s$-wave" case, it suffices to expand the Green function $G(q-$ $\left.p,-\varepsilon_{n}\right)$ to first order in hopping $t_{\perp}$. The evaluation of Eq. (76) with $\eta\left(\varphi_{p}\right)=\sqrt{2} \cos \left(2 \varphi_{p}\right)$ gives the same result as in the case of $s$-wave pairing with $\eta\left(\varphi_{p}\right)=1$. Then, the temperature functions of the AL diagram can be obtained from those for the $s$-wave pairing Eqs. (49) and (50) with the substitution of $\epsilon_{s}, \eta_{s}, \tau_{s}$, and $r_{s}$ by their $d$-wave analogs Eqs. (67)- (70).

In the clean limit we recover for $\kappa_{\alpha}^{(A L, L, d)}$ and $\kappa_{v}^{(A L, L, d)}$ their longitudinal $s$-wave, $T \tau$-independent analogs 15 . On the other hand, $\kappa^{(A L, L, d)}$ coefficients remain finite in the dirty limit. Moreover, in highly anisotropic materials at $r_{d} \ll \epsilon_{d}$, the AL contribution to the sound attenuation is clearly suppressed, and becomes important only at temperatures extremely close to $T_{c}$, just like the corresponding $s$-wave terms. The AL correction to the sound velocity is negligible just like the rMT one.

\section{Summary}

In summary, in the $d$-wave fluctuation corrections to longitudinal sound the aMT term is absent, and rMT and AL sound velocity corrections can be neglected in the range of parameters that is reasonable. The signs of the principal terms are as follows:

$$
\begin{aligned}
\Delta \alpha^{(D O S, T, s)} & <0, \quad \Delta \alpha^{(A L, T, s)}>0, \\
\Delta v^{(D O S, T, s)} & >0 .
\end{aligned}
$$

The magnitude of the corrections is finite though large in the clean limit, and it is suppressed to zero in the very dirty limit. Again, like in the $s$-wave case, the temperature dependence of the microscopic expressions for $d$-wave order parameter symmetry can be obtained in the phenomenological approach with the substitution of proper $\varepsilon_{d}, \eta_{d}$ and $r_{d}$ in the fluctuation term Eq. (17) and taking into account the strain dependence of $t_{\perp}$.

\section{Transverse phonons}

One can easily combine the results of Sec. $\mathrm{VB}$ and Sec. VIB

The DOS contribution is given by Eq. (52) with the fluctuation propagator modified according to Eq. (65). It is clear that after such a substitution one should obtain the results for the DOS longitudinal $d$-wave term because the angular dependence in $\left[\Gamma_{e p}^{(T)}\right]^{2}$ is averaged out.

Similar arguments are valid for the rMT contribution. Indeed, the longitudinal term contains the combination

$$
\begin{aligned}
& \Gamma_{e p}^{(L)}(\mathbf{p}) \Gamma_{e p}^{(L)}(\mathbf{q}-\mathbf{p}) \\
& =g_{L}^{2} \cos \left(p_{z} c\right) \cos \left[\left(q_{z}-p_{z}\right) c\right] .
\end{aligned}
$$

The corresponding combination in the transverse term at, for example, $\varphi=0$ can be rewritten to first nonvanishing order in the low-density limit $a p_{F} \ll 1$ :

$$
\begin{aligned}
& \Gamma_{e p}^{(T)}(\varphi=0, \mathbf{p}) \Gamma_{e p}^{(T)}(\varphi=0, \mathbf{q}-\mathbf{p}) \\
= & g_{T}^{2} \sin \left(p_{x} a\right) \sin \left(p_{z} c\right) \sin \left[\left(q_{x}-p_{x}\right) a\right] \sin \left[\left(q_{z}-p_{z}\right) c\right] \\
\approx & \tilde{g}_{T}^{2} \cos \left(q_{x} a\right) \cos ^{2}\left(\varphi_{p}\right) \sin \left(p_{z} c\right) \sin \left[\left(p_{z}-q_{z}\right) c\right]
\end{aligned}
$$

where $\left(a p_{F}\right)^{2}$ is absorbed in $\tilde{g}_{T}$, and the term linear in $\cos \left(\varphi_{p}\right)$ drops out after angular integration.

Since

$$
\begin{aligned}
& \int_{-\pi / c}^{\pi / c} \cos \left(p_{z} c\right) \cos \left[\left(q_{z}-p_{z}\right) c\right] d p_{z} \\
& \quad=-\int_{-\pi / c}^{\pi / c} \sin \left(p_{z} c\right) \sin \left[\left(q_{z}-p_{z}\right) c\right] d p_{z}=\frac{\pi}{c} \cos \left(q_{z} c\right),
\end{aligned}
$$

one can see that after integrating over $p_{z}$, the transverse term should contain an extra $\cos \left(q_{x} a\right) \cos ^{2}\left(\varphi_{p}\right)$ in comparison with the longitudinal one. Then, it suffices to set $\cos \left(q_{x} a\right) \approx 1$ at $q_{x} a \ll 1$, and $\cos ^{2}\left(\varphi_{p}\right)$ will provide an extra $1 / 2$ after the angular integration. We conclude that with the proper choice of the coupling constant, one can use the longitudinal $d$-wave results.

For the same reason as in the longitudinal $d$-wave case, there is no aMT contribution here.

Finally, as described in Sec. $\mathrm{\nabla B}$ in order to obtain non-zero $A L$ contribution, one should expand the Green function $G(q-p)$ either to second order in small $\Delta \xi(q, p)_{\perp}$ as in Eq. (57) (this results in non-singular AL contribution), or to linear order in $\Delta \xi(q, p)_{X}$ (this adds an additional small factor $\left(t_{X} / t_{\perp}\right)^{2}$ in front of the whole 
diagram). The details of the integration over $q$ are the same as in the transverse $s$-wave case. We conclude that the AL term can be neglected too.

The leading terms then read:

$$
\Delta \alpha^{(D O S, T, s)}<0, \quad \Delta v^{(D O S, T, s)}>0 .
$$

\section{DISCUSSION}

The superconducting fluctuations can provide a renormalization of the normal state sound attenuation and sound velocity that depends on the various phonon polarizations and order parameter symmetries. The magnitude of the effect, as can be seen from Eqs. (37) and (44), is of the order of $T_{c} / \varepsilon_{F}$ or less, depending on the material cleanness. For layered organics, the Fermi surface parameters of Ref. 32] lead to $T_{c} / \varepsilon_{F} \sim 10^{-2}$. The temperature functions $f^{(\beta, s)}\left(\epsilon, r, \gamma_{\phi}\right)$ increase this ratio at $T \rightarrow T_{c}$, thus making the fluctuation corrections experimentally measurable.

The actual sign of the fluctuation corrections is determined by the microscopic coefficients $\kappa_{\alpha}^{(\beta, \hat{e}, s)}$. We gather the information on signs and relative magnitudes of the microscopic fluctuation terms in Table I. In short, the leading contribution is always in the DOS channel, and there are comparable corrections from the aMT diagram only for $s$-wave attenuation but for both polarizations, and from the AL diagram only for longitudinal sound attenuation but for both symmetries. The rMT (always) and the AL (longitudinal sound velocity) temperature functions have a limit unity at $T=T_{c}$ but could compete with the leading terms only in the special cases of low anisotropy and short phase breaking time where the leading terms are suppressed. The aMT terms in the $s$-wave sound velocity and the AL terms for transverse phonon polarization contain additional small factors and can thus be neglected. Finally, there is no aMT term for $d$-wave symmetry of the order parameter because the diffusion pole disappears in this case.

The temperature dependence in the sound velocity obtained from the phenomenological Ginzburg-Landau free energy is in agreement with the microscopic calculations for the longitudinal phonons in both the $s$-wave and $d$ wave cases. In the latter case, it suffices to replace $\epsilon, \eta$ and $r$ by their $d$-wave equivalents $\epsilon_{d}, \eta_{d}$ and $r_{d}$ defined in Sec. VI] For example, one can compare the temperature dependence of the phenomenological result Eq. (19) with that of the DOS contribution in Eqs. (46) and (71). Also, the temperature dependence of the phenomenological Eq. (20) as well as the sign of the correction is the same as that of the rMT terms Eqs. (47) and (75). Similarly, the AL contributions (sign and temperature function) Eqs. (50) and Sec. VIB3 are explained by the phenomenological result Eq. (21). The Ginzburg-Landau approach does not treat the aMT contribution correctly because it does not include the dynamics involved in the diffusion.

In principle, one can obtain the fluctuation correc- tions to the sound velocity of transverse phonons from the Ginzburg-Landau approach as well. The modification will include taking into account the next-to-nearest neighbor hopping in the quasiparticle energy spectrum Eq. (16), extra hopping terms in the fluctuation free energy Eq. (11), and choosing the appropriate combination $\bar{c}$ in Eq. (5) that will modify the elastic terms Eq. (6).

It should be noted that the relation $v_{s \mathrm{fl}} \propto-C_{\mathrm{fl}}$ between fluctuation corrections to the sound velocity and specific heat that is valid in the bulk continuous model, is not satisfied in the tight-binding model with hopping terms in the electron quasiparticle energy spectrum. Thus, the experimental information from the specific heat measurements (first reference in [4]) is not enough to predict the behavior of the sound velocity fluctuation corrections in organic compounds.

We have obtained these results in the quasi-2D square lattice model for a corrugated cylindrical Fermi-surface. As a result, we see, for example, that there is no effect on the attenuation of the transverse sound as the direction of polarization is changed in the plane, both in the normal and in the superconducting states. In order to make a better fit of the experimental data, the generalization of our model to the particular crystal lattice (that is not tetragonal) and to the appropriate band structure is important. For example, it might be necessary to incorporate the dimer structure and triangular in-plane symmetry of organic materials. 29, 30, 31] That would better reflect the two-band energy spectrum of the $\kappa$ - $(\mathrm{ET})_{2} \mathrm{X}$ compounds. All these problems should be the subject of future research.

In conclusion, we have found how the sound attenuation and sound velocity are renormalized by superconducting fluctuations at temperatures close to $T_{c}$ in layered superconductors. For various polarizations of phonons propagating perpendicular to the conduction plane, we considered the cases of $s$ - and $d$-wave symmetry of the order parameter. In the hydrodynamic limit $\omega \tau \ll 1$ and $k \ell \ll 1$, we found the contributions from all the fluctuation diagrams (namely, the AL, MT and DOS) and provided the theoretical background for the analysis of the experimental information in organic superconductors. The complete analytical expressions for the microscopic fluctuation corrections can be found elsewhere [33].

\section{ACKNOWLEDGEMENTS}

The authors would like to thank K.V. Samokhin and M. Walker for valuable discussions, and D. Fournier, C. Lupien, M. Poirier, and L. Taillefer for information on recent experiments in the field. The present work was supported by the Natural Sciences and Engineering Research Council (NSERC) of Canada, the Fonds de la Recherche sur la Nature et les Technologies of the Québec government, and the Tier I Canada Research Chair Program (A.-M.S.T.). 


\begin{tabular}{|c|c|c|c|c|c|c|}
\hline \hline symmetry & mode & channel & DOS & rMT & aMT & AL \\
\hline \multirow{3}{*}{$s$} & $\mathrm{~L}[001]$ & $\alpha$ & - & $\lesssim|+1|$ & - & + \\
& & $v_{s}$ & + & $\lesssim|-1|$ & $O\left(\omega^{2}\right)$ & $\lesssim|-1|$ \\
& $\mathrm{T}[001]$ & $\alpha$ & - & $\lesssim|+1|$ & - & $O\left(\left(t_{X} / t_{\perp}\right)^{2}\right)$ \\
& & $v_{s}$ & + & $\lesssim|-1|$ & $O\left(\omega^{2}\right)$ & $O\left(\left(t_{X} / t_{\perp}\right)^{2}\right)$ \\
\hline \multirow{4}{*}{$d$} & \multirow{2}{*}[001]{} & $\alpha$ & - & $\lesssim|+1|$ & none & + \\
& & $v_{s}$ & + & $\lesssim|-1|$ & none & $\lesssim|-1|$ \\
& $\mathrm{T}[001]$ & $\alpha$ & - & $\lesssim|+1|$ & none & $O\left(\left(t_{X} / t_{\perp}\right)^{2}\right)$ \\
& & $v_{s}$ & + & $\lesssim|-1|$ & none & $O\left(\left(t_{X} / t_{\perp}\right)^{2}\right)$ \\
\hline \hline
\end{tabular}

TABLE I: Summary of the fluctuation contributions from different diagrams for various order parameter symmetries and phonon polarizations. The symbol $\lesssim|-1|$ means that the sign of the correction is negative and that the absolute value of the temperature function is less than unity with limit unity at $T=T_{c}$.

[1] C. Lupien, W.A. MacFarlane, C. Proust, L. Taillefer, Z.Q. Mao, and Y. Maeno, Phys. Rev. Lett. 86, 5986 (2001).

[2] B.S. Shivaram et. al, Phys. Rev. Lett. 56, 1078 (1986); B. Battlog et. al, Phys. Rev. Lett. 55, 1319 (1985); B. Goding et. al, ibid. 55, 2479 (1985).

[3] K. Frikach, M. Poirier, M. Castonguay, and K.D. Truong Phys. Rev. B 61, R6491 (2000); D. Fournier, M. Poirier, M. Castonguay, and K. Truong, Phys. Rev. Lett. 90, 127002 (2003).

[4] J.E. Graebner, R.C. Haddon, S.V. Chichester, and S.H. Glarum, Phys. Rev. B 41, 4808 (1990); D.E. Farrell, C.J. Allen, R.C. Haddon, and S.V. Chichester, Phys. Rev. B 42, 8694 (1990); M. Lang, F. Steglich, N. Toyota, and T. Sasaki, Phys. Rev. B 49, 15227 (1994); S. Friemel, C. Pasquier, Y. Loirat, and D. Jérome, Physica C: Superconductivity 259, 181 (1996); S. Belin, K. Behnia, and A. Deluzet, Phys. Rev. Lett. 81, 4728 (1998); N.J. Clayton, H. Ito, S.M. Hayden, P.J. Meeson, M. Springford, and G. Saito, Phys. Rev. B 65, 064515 (2002).

[5] K. Frikach, PhD thesis, Université de Sherbrooke 2001 (unpublished).

[6] A. Schmid, Z. Physik 259, 421 (1973).

[7] A.B. Pippard, Philosophical magazine 46, 1104 (1955).

[8] T. Tsuneto, Phys. Rev 121, 402 (1961).

[9] M.B. Walker, M.F. Smith, and K.V. Samokhin, Phys. Rev. B 65, 014517 (2002).

[10] J. Moreno, and P. Coleman, Phys. Rev. B 53, R2995 (1996).

[11] P. Contreras, M. Walker, and K. Samokhin, cond-mat/0407181

[12] L.G. Aslamazov and A.I. Larkin, Soviet Solid State 10, 875 (1968); Phys. Lett. 26A, 238 (1968).

[13] K. Maki, Progr. Theor. Phys. (Kyoto) 39, 897 (1968); 40, 193 (1968).

[14] R.S. Thompson, Phys. Rev. B 1, 327 (1970).

[15] M.S. Mar'enko, C. Bourbonnais, and A.-M.S. Tremblay, Phys. Rev. B 69, 224503 (2004).

[16] L.D. Landau, and E.M. Lifshitz, "Theory of Elasticity", volume 7 of "Course of Theoretical Physics ", Pergamon Press Ltd., London (1959).

[17] N.W. Ashcroft, N.D. Mermin, "Solid State Physics", W.B. Saunders Company, (1976).
[18] L.R. Testardi, Phys. Rev. B 3, 95 (1971).

[19] A.I. Larkin and A.A. Varlamov, Fluctuation Phenomena in Superconductors, in "Handbook on Superconductivity: Conventional and Unconventional Superconductors", ed. by K.-H. Bennemann and J.B. Ketterson, Springer, (2002).

[20] See Sec. 147 of L.D. Landau, and E.M. Lifshitz, "Statistical Physics", volume 5 of "Course of Theoretical Physics", Pergamon Press Ltd., London (1959).

[21] J.M. Caulfield, W. Lubczynski, F.L. Pratt, J. Singleton, D.Y.K. Ko, W. Hayes, M. Kurmoo, and P. Day, J. Phys.: Condens. Matter 6, 2911 (1994); John Singleton, P.A. Goddard, A. Ardavan, N. Harrison, S.J. Blundell, J.A. Schlueter, and A.M. Kini, Phys. Rev. Lett. 88, 037001 (2002).

[22] J.J. McGuire, T. Rõõm, A. Pronin, T. Timusk, J.A. Schueter,M.E. Kelly, and A.M. Kini, Phys. Rev. B 64, 094503 (2001).

[23] P. Moses and R.H. McKenzie, Phys. Rev. B 60, 7998 (1999)

[24] A.A. Abrikosov, L.P. Gorkov, and I.E. Dzyaloshinski, "Methods of the quantum field theory in statistical physics", Dover Publications, New York (1963).

[25] A.A. Abrikosov, "Introduction to the Theory of Normal Metals", Academic Press, New York (1972).

[26] R.A. Klemm, M.R. Beasley, and A. Luther, Phys. Rev. B 8, 5072 (1973); R.A. Klemm, J. Low Temp. Phys. 16, 381 (1974).

[27] S. Yip, Phys. Rev. B 41, 2612 (1990). P. Carretta, A. Rigamonti, A.A. Varlamov, and D.V. Livanov, Phys. Rev. B 54, R9682 (1996).

[28] M. Eschrig, D. Rainer, and J.A. Sauls, Phys. Rev. B 59, 12095 (1999).

[29] J.M. Williams et al., Science 252, 1501 (1991); D. Jerome, ibid. 252, 1509 (1991).

[30] H. Kino, and H. Fukuyama, J. Phys. Soc. Jpn. 65, 2158 (1996).

[31] R.H. McKenzie, Comments Cond. Matt. Phys. 18, 309 (1998).

[32] M. Dressel, O. Klein, G. Grüner, K.D. Carlson, H.H. Wang, and J.M. Williams, Phys. Rev. B 50, 13603 (1994).

[33] The Mathematica source files as well as .pdf version for the fluctuation corrections can be found at 
http://www.physique.usherbrooke.ca/tremblay/pub/sound_unconv.htm 